\title{
A Bibliography of Spiking Neural P Systems
}

\author{
Gheorghe Păun \\ Institute of Mathematics of the Romanian Academy \\ PO Box 1-764, 014700 Bucureşti, Romania \\ and \\ Department of Computer Science and Artificial Intelligence \\ University of Sevilla \\ Avda. Reina Mercedes s/n, 41012 Sevilla, Spain \\ george.paun@imar.ro, gpaun@us.es
}

What follows is a bibliography of spiking neural $\mathrm{P}$ systems (SN P systems, for short), at the level of April 2009. This bibliography is included in the present volume having in mind the fact that this research area attracted much interest in the few years since it was initiated, so that it might be useful to the reader to have a comprehensive list of titles at hand, as complete as we were able to compile it. Of course, most of this information can also be found in the membrane computing website, at http://ppage.psystems.eu, with a comprehensive survey also provided by the chapter devoted to SN P systems in the Handbook of Membrane Computing (Gh. Păun, G. Rozenberg, A. Salomaa, eds.), Oxford University Press, 2009. The list which follows also includes the papers on SN P systems present in the present volume (with the indication "in the present volume"); the papers from the previous proceedings volumes of the Brainstorming Week on Membrane Computing are given with indications of the form "BWMC2007" with the obvious meaning, then specifying the pages. The three brainstorming volumes cited in this way are:

1. M.A. Gutiérrez-Naranjo, et al., eds.: Fourth Brainstorming Week on Membrane Computing, Sevilla, January 30-February 3, 2006, vol. I and II, Fenix Editora, Sevilla, 2006.

2. M.A. Gutiérrez-Naranjo, et al., eds.: Fiftth Brainstorming Week on Membrane Computing, Sevilla, January 29-February 2, 2007, Fenix Editora, Sevilla, 2007.

3. D. Diaz-Pernil, et al., eds.: Sixth Brainstorming Week on Membrane Computing, Sevilla, February 4-8, 2008, Fenix Editora, Sevilla, 2008.

For some of the papers it is indicated only the year, this meaning that the information we had when compiling the list was that the paper was still unpublished. 
1. A. Alhazov, R. Freund, M. Oswald, M. Slavkovik: Extended variants of spiking neural $\mathrm{P}$ systems generating strings and vectors of non-negative integers. WMCr, 2006, 88-101, and Membrane Computing, WMC2006, Leiden, Revised, Selected and Invited Papers, LNCS 4361, Springer, 2006, 123-134.

2. A. Binder, R. Freund, M. Oswald: Extended spiking neural P systems with astrocytes - variants for modelling the brain. Proc. 13th Intern. Symp. AL and Robotics, AROB2008, Beppu, Japan, 520-524.

3. A. Binder, R. Freund, M. Oswald, L. Vock: Extended spiking neural P systems with excitatory and inhibitory astrocytes. Submitted, 2007.

4. M. Cavaliere, E. Egecioglu, O.H. Ibarra, M. Ionescu, Gh. Păun, S. Woodworth: Asynchronous spiking neural $\mathrm{P}$ systems; decidability and undecidability. Proc. DNA13, LNCS 4848, Springer, 2007.

5. M. Cavaliere, E. Egecioglu, O.H. Ibarra, M. Ionescu, Gh. Păun, S. Woodworth: Asynchronous spiking neural P systems. Theoretical Computer Sci., 2009.

6. M. Cavaliere, I. Mura: Experiments on the reliability of stochastic spiking neural P systems. Natural Computing, 7, 4 (2008), 453-470.

7. R. Ceterchi, A.I. Tomescu: Spiking neural $\mathrm{P}$ systems - a natural model for sorting networks. BWMC2008, 93-106.

8. H. Chen, R. Freund, M. Ionescu, Gh. Păun, M.J. Pérez-Jiménez: On string languages generated by spiking neural $\mathrm{P}$ systems. BWMC2006, vol. I, 169194, and Fundamenta Informaticae, 75, 1-4 (2007), 141-162.

9. H. Chen, M. Ionescu, T.-O. Ishdorj: On the efficiency of spiking neural P systems. BWMC2006, vol. I, 195-206, and Proc. 8th Intern. Conf. on Electronics, Information, and Communication, Ulanbator, Mongolia, June 2006, 49-52.

10. H. Chen, M. Ionescu, T.-O. Ishdorj, A. Păun, Gh. Păun, M.J. Pérez-Jiménez: Spiking neural $\mathrm{P}$ systems with extended rules: Universality and languages. Natural Computing, 7, 2 (2008), 147-166.

11. H. Chen, M. Ionescu, A. Păun, Gh. Păun, B. Popa: On trace languages generated by spiking neural P systems. BWMC2006, vol. I, 207-224, and Eighth International Workshop on Descriptional Complexity of Formal Systems (DCFS 2006), June 21-23, 2006, Las Cruces, New Mexico, USA, 94-105.

12. H. Chen, T.-O. Ishdorj, Gh. Păun: Computing along the axon. BWMC2006, vol. I, 225-240, and Pre-proceedings BIC-TA, Wuhan, 2006, 60-70, and Progress in Natural Science, 17, 4 (2007), 418-423.

13. H. Chen, T.-O. Ishdorj, Gh. Păun, M.J. Pérez-Jiménez: Spiking neural P systems with extended rules. BWMC2006, vol. I, 241-266.

14. H. Chen, T.-O. Ishdorj, Gh. Păun, M.J. Pérez-Jiménez: Handling languages with spiking neural $\mathrm{P}$ systems with extended rules. Romanian J. Information Sci. and Technology, 9, 3 (2006), 151-162.

15. R. Freund, M. Ionescu, M. Oswald: Extended spiking neural P systems with decaying spikes and/or total spiking. ACMC/FCT 2007 Workshop, Budapest, Intern. J. Found. Computer Sci., 19 (2008), 1223-1234.

16. R. Freund, M. Oswald: Spiking neural P systems with inhibitory axons. $A R O B$ Conf., Japan, 2007. 
17. R. Freund, M. Oswald: Regular $\omega$-languages defined by extended spiking neural P systems. Fundamenta Informaticae, 83, 1-2 (2008), 65-73.

18. M. Garcia-Arnau, D. Pérez, A. Rodriguez-Patón, P. Sosik: On the power of elementary operations in spiking neural P systems. Submitted, 2008.

19. M. Garcia-Arnau, A. Rodriguez-Patón, D. Pérez, P. Sosik: Spiking neural P systems: Stronger normal forms. BWMC200\%, 157-178.

20. M.A. Gutiérrez-Naranjo, A. Leporati: Solving numerical NP-complete problems by spiking neural P systems with pre-computed resources. BWMC2008, $193-210$.

21. M.A. Gutiérrez-Naranjo, A. Leporati: Performing arithmetic operations with spiking neural $\mathrm{P}$ systems. In the present volume.

22. M.A. Gutiérrez-Naranjo, M.J. Pérez-Jiménez. A first model for Hebbian learning with spiking neural P systems. BWMC2008, 211-234.

23. O.H. Ibarra, A. Păun, Gh. Păun, A. Rodriguez-Patón, P. Sosik, S. Woodworth: Normal forms for spiking neural P systems. BWMC2006, vol. II, 105-136, and Theoretical Computer Sci., 372, 2-3 (2007), 196-217.

24. O.H. Ibarra, A. Păun, A. Rodriguez-Patón: Sequentiality induced by spike numbers in SN P systems. Proc. 14th Intern. Meeting on DNA Computing, Prague, June 2008, 36-46.

25. O.H. Ibarra, S. Woodworth: Characterizations of some restricted spiking neural P systems. and Membrane Computing, WMC2006, Leiden, Revised, Selected and Invited Papers, LNCS 4361, Springer, 2006, 424-442.

26. O.H. Ibarra, S. Woodworth: Spiking neural P systems: some characterizations. Proc. FCT 2007, Budapest, LNCS 4639, 23-37.

27. O.H. Ibarra, S. Woodworth: Characterizing regular languages by spiking neural P systems. Intern. J. Found. Computer Sci., 18, 6 (2007), 1247-1256.

28. O.H. Ibarra, S. Woodworth, F. Yu, A. Păun: On spiking neural P systems and partially blind counter machines. Proc. UC2006, LNCS 4135, Springer, 2006, $113-129$

29. M. Ionescu, A. Păun, Gh. Păun, M.J. Pérez-Jiménez: Computing with spiking neural P systems: Traces and small universal systems. Proc. DNA12 (C. Mao, Y. Yokomori, B.-T. Zhang, eds.), Seul, June 2006, 32-42, and DNA Computing. 12th Intern. Meeting on DNA Computing, DNA12, Seoul, Korea, June 2006, Revised Selected Papers (C. Mao, T. Yokomori, eds.), LNCS 4287, Springer, 2007, 1-16.

30. M. Ionescu, Gh. Păun, T. Yokomori: Spiking neural P systems. Fundamenta Informaticae, 71, 2-3 (2006), 279-308.

31. M. Ionescu, Gh. Păun, T. Yokomori: Spiking neural P systems with an exhaustive use of rules. Intern. J. Unconventional Computing, 3, 2 (2007), 135-154.

32. M. Ionescu, D. Sburlan: Some applications of spiking neural P systems. Proc. WMC8, Thessaloniki, June 2007, 383-394, and Computing and Informatics, 27 (2008), 515-528.

33. M. Ionescu, C.I. Tîrnăucă, C. Tîrnăucă: Dreams and spiking neural P systems. Romanian J. Inform. Sci. and Technology, 12 (2009), in press. 
34. T.-O. Ishdorj, A. Leporati: Uniform solutions to SAT and 3-SAT by spiking neural P systems with pre-computed resources. Natural Computing, to appear.

35. T.-O. Ishdorj, A. Leporati, L. Pan, X. Zeng, X. Zhang: Deterministic solutions to QSAT and Q3SAT by spiking neural P systems with pre-computed resources. In the present volume.

36. A. Leporati, G. Mauri, C. Zandron, Gh. Păun, M.J. Pérez-Jiménez: Uniform solutions to SAT and Subset-Sum by spiking neural P systems. Submitted, 2007.

37. A. Leporati, C. Zandron, C. Ferretti, G. Mauri: On the computational power of spiking neural P systems. BWMC2007, 227-246.

38. A. Leporati, C. Zandron, C. Ferretti, G. Mauri: Solving numerical NP-complete problems with spiking neural $\mathrm{P}$ systems. Proc. WMC8, Thessaloniki, June 2007, 405-424.

39. V.P. Metta, K. Krithivasan: Spiking neural P systems and Petri nets. Submitted, 2008.

40. J.M. Mingo: Una approximacion al control neural del sueno de ondas lentas mediante spiking neural P systems. Submitted, 2008.

41. J.M. Mingo: Sleep-awake switch with spiking neural P systems: A basic proposal and new issues. In the present volume.

42. T. Neary: A small universal spiking neural P system. Intern. Workshop. Computing with Biomolecules (E. Csuhaj-Varju et al., eds.), Viena, 2008, 65-74.

43. T. Neary: On the computational complexity of spiking neural P systems. Unconventional Computation. 7th Intern. Conf. Vienna, 2008 (C.S. Calude at al., eds.), LNCS 5204, 2008, 189-205.

44. A. Obtulowicz: Spiking neural P systems and modularization of complex networks from cortical neural network to social networks. In the present volume.

45. L. Pan, Gh. Păun: New normal forms for spiking neural P systems. In the present volume.

46. L. Pan, Gh. Păun: Spiking neural P systems with anti-spikes. In the present volume.

47. L. Pan, Gh. Păun, M.J. Pérez-Jiménez: Spiking neural P systems with neuron division and budding. In the present volume.

48. L. Pan, Gh. Păun, M.J. Pérez-Jiménez: Spiking neural P systems: A short introduction and new normal forms, Advanced Computational Tehcnologies (C. Enăchescu, F. Filip, B. Iantovics, eds.), Ed. Academiei, Bucureşti, 2009.

49. L. Pan, J. Wang, H.J. Hoogeboom: Excitatory and inhibitory neural P systems. Submitted, 2007.

50. A. Păun, Gh. Păun: Small universal spiking neural P systems. BioSystems, 90, 1 (2007), 48-60.

51. Gh. Păun: Languages in membrane computing. Some details for spiking neural P systems. Proc. 10th DLT Conf. (invited talk), Santa Barbara, USA, 2006, LNCS 4036, Springer, Berlin, 2006, 20-35.

52. Gh. Păun: Twenty six research topics about spiking neural $\mathrm{P}$ systems. BWMC2007, 263-280. 
53. Gh. Păun: A quick overview of membrane computing with some details about spiking neural P systems. Frontiers of Computer Science in China, 1,1 (2007), 37-49.

54. Gh. Păun: Spiking neural P systems. A tutorial. Bulletin of the EATCS, 91 (Febr. 2007), 145-159.

55. Gh. Păun: Spiking neural P systems. Power and efficiency. Bio-Inspired Modeling of Cognitive Tasks, Proc. IWINAC 2007 (J. Mira, J.R. Alvarez, eds.), Mar Menor, 2007, LNCS 4527, 153-169.

56. Gh. Păun: Spiking neural $\mathrm{P}$ systems used as acceptors and transducers. CIAA 2007, 12th Conf., Prague, July 2007, LNCS 4783 (J. Holub, J. Zdarek, eds.), Springer, Berlin, 2007, 1-4.

57. Gh. Păun: Spiking neural P systems with astrocyte-like control. JUCS, 13, 11 (2007), 1707-1721.

58. Gh. Păun, M.J. Pérez-Jiménez: Spiking neural P systems. Recent results, research topics. Submitted, 2007.

59. Gh. Păun, M.J. Pérez-Jiménez: Spiking neural P systems. An overview. Advancing Artificial Intelligence through Biological Process Applications (A.B. Porto, A. Pazos, W. Buno, eds.), Medical Information Science Reference, Hershey, New York, 2008, 60-73.

60. Gh. Păun, M.J. Pérez-Jiménez, G. Rozenberg: Spike trains in spiking neural P systems. Intern. J. Found. Computer Sci., 17, 4 (2006), 975-1002.

61. Gh. Păun, M.J. Pérez-Jiménez, G. Rozenberg: Computing morphisms by spiking neural P systems. Intern. J. Found. Computer Sci., 18, 6 (2007), 13711382 .

62. Gh. Păun, M.J. Pérez-Jiménez, G. Rozenberg: Infinite spike trains in spiking neural P systems. Manuscript, 2005.

63. Gh. Păun, M.J. Pérez-Jiménez, A. Salomaa: Bounding the indegree of spiking neural P systems. TUCS Technical Report 773, 2006.

64. Gh. Păun, M.J. Pérez-Jiménez, A. Salomaa: Spiking neural P systems. An early survey. Intern. J. Found. Computer Sci., 18 (2007), 435-456.

65. D. Ramirez-Martinez, M.A. Gutiérrez-Naranjo: A software tool for dealing with spiking neural P systems. BWMC200\%, 299-314.

66. J. Wang, T.-O. Ishdorj, L. Pan: Efficiency of spiking neural P systems. In the present volume.

67. X. Zeng, X. Zhang, L. Pan: Homogeneous spiking neural P systems. Submitted, 2009.

68. X. Zhang, T.-O. Ishdorj, X. Zeng, L. Pan: Solving PSPACE-complete problems by spiking neural P systems with pre-computed resources. Submitted, 2008.

69. X. Zhang, Y. Jiang, L. Pan: Small universal spiking neural P systems with exhaustive use of rules. Proc. Third Intern. Conf. on Bio-Inspired Computing. Theory and Appl., Adelaide, 2008, 117-127.

70. X. Zhang, J. Wang, L. Pan: A note on the generative power of axon $\mathrm{P}$ systems. Intern. J. CCC, 4, 1 (2009), 92-98. 
71. X. Zhang, X. Zeng, L. Pan: On string languages generated by SN P systems with exhaustive use of rules. Natural Computing, 7 (2008), 535-549.

72. X. Zhang, X. Zeng, L. Pan: Smaller universal spiking neural P systems. Fundamenta Informaticae, 87 (2008), 117-136.

73. X. Zhang, X. Zeng, L. Pan: On string languages generated by asynchronous spiking neural $\mathrm{P}$ systems. Theoretical Computer Science, DOI: 10.1016/j.tcs.2008.12.055. 